\title{
DESPECKLING OF SAR IMAGES BY OPTIMIZING AVERAGED POWER SPECTRAL VALUE IN CURVELET DOMAIN
}

\author{
S.Md.Mansoor Roomi $^{1 \#}$, D.Kalaiyarasi ${ }^{2 \#}$ \\ \#Department of ECE, Thiagarajar College of Engineering, Madurai-15, TamilNadu,India \\ ${ }^{1}$ smmroomi@tce.edu, ${ }^{2} \mathrm{kal}$ aiecedurai@gmail.com
}

\begin{abstract}
Synthetic Aperture Radar (SAR) images are inherently affected by multiplicative speckle noise, due to the coherent nature of scattering phenomena. In this paper, a novel algorithm capable of suppressing speckle noise using Particle Swarm Optimization (PSO) technique is presented. The algorithm initially identifies homogenous region from the corrupted image and uses PSO to optimize the Thresholding of curvelet coefficients to recover the original image. Average Power Spectrum Value (APSV) has been used as objective function of PSO. The Proposed algorithm removes Speckle noise effectively and the performance of the algorithm is tested and compared with Mean filter, Median filter, Lee filter, Statistic Lee filter, Kuan filter, frost filter and gamma filter., outperforming conventional filtering methods.
\end{abstract}

\section{KEYWORDS}

Curvelet, Speckle noise, SAR images, Thresholding techniques, speckle filters, Particle Swam Optimization, Soft thresholding, Fourier transform, Average power spectrum.

\section{INTRODUCTION}

The Speckle in the SAR images reduces the detection ability of targets and is not favourable to the image understanding [1]. Thus, the despeckling has become an important issue in SAR image processing. Despeckling can be carried out in the spatial domain, such as Median filter[2], Lee filter [6], Statistical Lee filter[7], Kuan filter [8], Frost filter [9],Gamma MAP filter[10] are among the better denoising algorithms in radar community. But the despeckling efficiency of these filters are proportional to the size of the window, and they may blur the details of the image when the window is too big.

Recent years, wavelet theory has become one of the main tools of the signal processing. It's analysis capacity for the time domain and frequency domain of the signal and optimal approximation to one-dimensional bounded variable function classes is the main reason that wavelet develops so rapidly [4]. To one dimensional signals which include singular points, wavelet can meet "optimal" non linear approximation order, but because of the two dimensional wavelet transform is isotropic, and when dealing with two dimensions or more dimensions signals which include singular lines, transform coefficients of the local maximum module can only reflect the position that the wavelet coefficients turn up "pass" the edge but cannot express the information "along" the edge. So it turns out some limitation [11]. 
International Journal of Information Sciences and Techniques (IJIST) Vol.2, No.2, March 2012

Due to the above mentioned shortcomings of wavelet transform. Donoho and others proposed Curvelet transform theory and their anisotropy characters is very useful for the efficient expression of the image edge and get good results in image denoising [20]. There two fast implementations of the curvelet transform which are faithful to mathematical transformation, one via USFFT the other via wrapping, the later is used in this paper [5].

In this paper, Section II explains about the speckle Noise model, Section III describes about curvelet Transform, Section IV describes about proposed algorithm, and Section V provides the Results and Discussion of the proposed method. A brief Conclusion is given in Section VI.

\section{SPECKLE NOISE MODEL}

Speckle noise in SAR images is usually modeled as a stationary multiplicative noise with unit mean and variance [3]. A simple model for speckle noisy image has a multiplicative is represented by

$$
f(x, y)=S(x, y) \cdot N(x, y)
$$

By applying logarithmic operator to both sides of eqn (1), the following expression is obtained

$$
\ln (Y)=\ln (S)+\ln (N)
$$

Equation (2) can be written as

$$
y(x, y)=s(x, y)+e(x, y)
$$

Where $\mathrm{y}(\mathrm{x}, \mathrm{y}), \mathrm{s}(\mathrm{x}, \mathrm{y})$ and $\mathrm{e}(\mathrm{x}, \mathrm{y})$ represent the logarithmically transformed noisy data, signal and speckle noise respectively. This nonlinear transform totally changes the statistics of speckle noise. Pixels of log-transformed images are mutually independent and this makes less difficult to extract information from speckled images.

\section{CURVELET TRANSFORM}

Curvelet transform (CT) is proposed by Candes and Donoho in 1999, its essence is derived from the ridge-wave theory [13][14]. In the foundation of single ridge-wave or local ridge wave transform, Curvelet transform is constructed to express the objects which have curved singular boundary, curvelet combines advantages of ridge wave which is suitable for expressing the points character and wavelet which is suitable for expressing the points character and take full advantage of multi scale analysis, it is suitable for large class of image processing problems.

The Curvelet transform of function is written as,

$$
c(j, l, k):=\left\langle f, \varphi_{i j, l, k}\right\rangle
$$

Among which, $\varphi_{i j, l, k}$ is Curvelet coefficient, $j, l, k$ are the parameters of scale. Direction and position respectively. Wrapping round origin is the core of Wrapped based curvelet. It realizes one to one through the periodization technology in the affine region. Fast Digital Curvelet Transform via wrapping as follows [16]:

1. Apply the 2D FFT and obtain fourier sample $\hat{f}\left[n_{1}, n_{2}\right],-n / 2 \leq n_{1}, n_{2}<n / 2$ 
International Journal of Information Sciences and Techniques (IJIST) Vol.2, No.2, March 2012

2. For each scale $\mathrm{j}$ and angle $l$, form the product $\tilde{U}_{j, l}\left[n_{1}, n_{2}\right] \hat{f}\left[n_{1}, n_{2}\right]$

3. Wrap this product the origin and obtain

$$
\tilde{f}_{j, l}\left[n_{1}, n_{2}\right]=W\left(\tilde{U}_{j, l}\left[n_{1}, n_{2}\right]\right) \hat{f}\left[n_{1}, n_{2}\right]
$$

Where the range for $n_{1}$ and $n_{2}$ is now $0 \leq n_{1}<L_{1, j}$ and $0 \leq n_{1}<L_{2, j}$ (for $\theta$ in the range $(\Pi / 4,-\Pi / 4)$ )

4. Apply the inverse 2D FFT to each $\tilde{f}_{j, l}$, hence collecting the discrete coefficients $\mathrm{C}^{\mathrm{D}}(j, l, k)$

5. Practice has proved that traditional curvelet transform method has the phenomenon that it would appear slightly "Scratches" and "ringing" in the image which is dialed with by denoising and reconstruction.

\section{Proposed Method}

Corrupted image is initially divided into blocks and for each block variance is calculated to identify homogenous block in the image. In the uniform block signal variance will be very low compared with non uniform blocks in the image. Variance describes how far the values lie from mean. Low variance block is identified with their positions. The Flow chart of the proposed algorithm of is shown in (Fig .1).Particle Swarm optimization is initiated to provide threshold value for despeckling the image in the curvelet domain. The position of the minimum variance block found initially is used for each iteration of PSO [12]. The PSO tends to minimize the average power spectrum value of block the position obtained from the previous stage. This is found as the error between successive variance of the uniform block. The PSO is terminated when the error variance is zero or within a tolerable limit, thus providing a despeckled image 


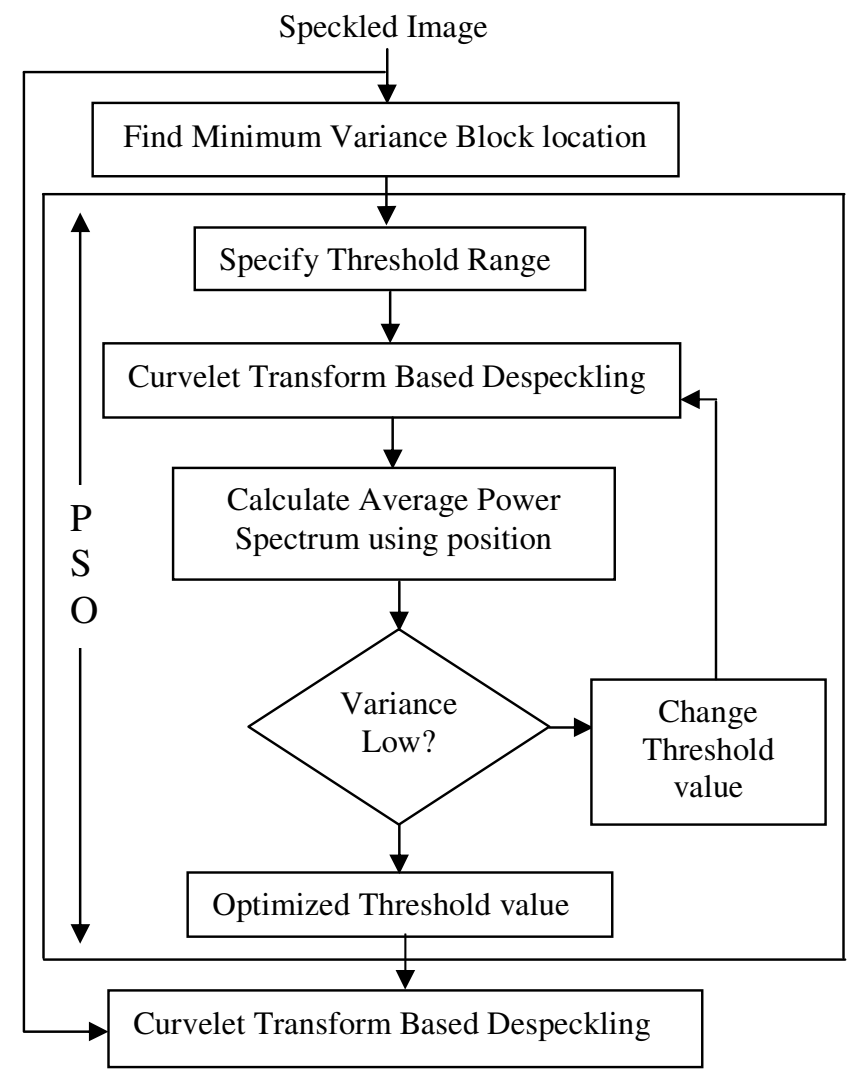

Figure.1. Flow Chart of the Proposed Method

\subsection{Variance Calculation}

Image is divided into $32 \times 32$ non-overlapping blocks and for each block the variance is calculated. Variance describes how far the current values are lie from mean. Variance is calculated using the formula,

$$
V=\frac{1}{R C} \sum_{i=1}^{R} \sum_{j=1}^{C} B_{i j}-\mu_{B}
$$

Where $\mu_{\mathrm{B}}$ is mean of the Block and $\mathrm{R}, \mathrm{C}$ are sizes of the Block.

\subsection{Average Power Spectrum Value (APSV)}

Transforming image into frequency domain can analyse characteristics of the signal [19]. For a homogenous region pixel gray values are same, thus it contains low frequency component and lesser power spectrum value. The presence of noise will increase frequency component of the signal hence leads to increased average power spectrum value. For the image $f=\{f[i, j] ; 0 \leq i, j \leq M-1\}$, differential image is obtained using $g[i, j]=f[i, j]-f[i, j-1], 0 \leq i, j \leq M-1$; translate the two diamensional differential signal into a one-diamensional signals $s[M i+j]=g[i, j], 0 \leq i, j \leq M-1$; Average power Spectrum value estimation of signal as follows: 
International Journal of Information Sciences and Techniques (IJIST) Vol.2, No.2, March 2012

1. Identified minimum variance block is divided into $\mathrm{k}$ segments

$$
x^{(k)}=\left\{x[n]=s\left[n_{k}+n\right] ; 0 \leq n \leq N-1\right\}
$$

2. Obtain $x^{(k)}$ FFT transform, $x^{(k)}=\{x(l) ; 0 \leq n \leq N-1\}$

3. The Power spectrum of each segment can be estimated as

$$
P^{(k)}=\left\{P^{(k)}(l) ; 0 \leq l \leq N / 2\right\}
$$

4. The power spectrum of the $L$ segments is estimated as:

$$
P[l]=\frac{1}{L} \sum_{k=1}^{L} p^{x}[l] ; 0 \leq l \leq N / 2
$$

\subsection{Curvelet Transform Based Despeckling}

Apply the fdct-wrapping to the log transformed image to obtain multi-resolution and multi directional coefficients $\mathrm{C}\{\mathrm{j}\}\{l\}$ where $\mathrm{j}$ is scale index and $\mathrm{j}=1,2, . .5, l$ is direction index. All of the coefficients in the finest scale $\mathrm{C}\{5\}\{1\}$ are set to zero. In additive noise condition we can get the shrinkage of the curvelet coefficient. Curvelet Transform based despeckling is shown in figure. 2 for every coefficient $\mathrm{C}(\mathrm{i}, \mathrm{j})$ within $\mathrm{C}\{\mathrm{j}\}\{l\}$, if $|\mathrm{C}(\mathrm{i}, \mathrm{j})|>\mathrm{T}$, apply a $3 \mathrm{X} 3$ window to $\mathrm{C}(\mathrm{i}, \mathrm{j})$, when there are more than two coefficients whose absolutely value is bigger than $\mathrm{T}$ in this window, these coefficients are marked as one, the others are marked as zero. After all the coefficients in $\mathrm{C}\{\mathrm{j}\}\{l\}$ are marked, if $\mathrm{C}(\mathrm{i}, \mathrm{j})$ is marked as one, it remains unchanged, or it shrinks based on threshold return by PSO. Then reconstructed image is obtained using inverse fdct-wrapping. Finally Antilogarithmic Transform is applied to get the despeckled image.

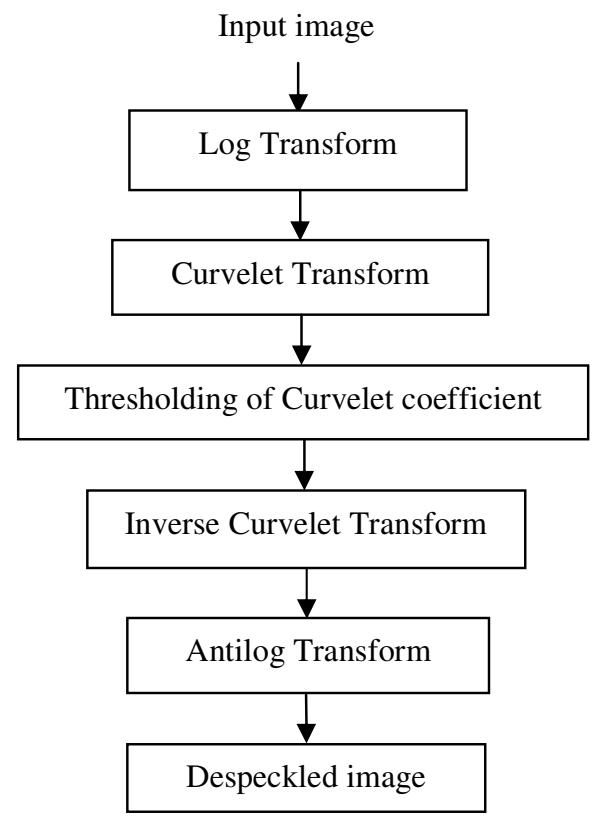

Figure.2. Flow Chart of Curvelet Transform Based Despeckling

\subsection{Particle Swarm Optimization}

PSO was first proposed by Eberhart and Kennedy [15].This technique is a population based optimization problem solving algorithm. Specify the parameters in PSO such as population size $(n)$, upper and lower bound values of problem space(xlow, xhigh), fitness function $(J)$, maximum 
and minimum velocity of particles (Vmax and Vmin, respectively), maximum and minimum inertia weights (Qmax and Qmin, respectively).

1. Initialize $n$ particles with random positions within upper and lower bound values of the problem space.

2. Evaluate the fitness function $(\mathrm{J})$ of Average power spectrum value for each particle using the detected uniform area from the initial iterated image. For each particle, find the best position found by particle $i$ call it Xpi and let the fitness value associated with it be $J p b e s t i$. At first iteration, position of each particle and its fitness value of $i t h$ particle are set to Xpi and Jpbesti, respectively.

3. Find a best position found by swarm call it $\mathrm{G}$ which is the position that maximum fitness value is obtained. Let the fitness value associated with it be JGbest. To find $\mathrm{G}$ the following algorithm described by pseudo code is adopted.

(At Initial iteration $\mathrm{n}$ set Jgbest $=0$ )

$$
\begin{aligned}
& \text { For } i=1 \text { to } n \text { do } \\
& \text { If Jpbesti }>\text { Jgbest, then } \\
& \quad G=\text { Xpi, JGbest= Jpbest } \\
& \text { end; }
\end{aligned}
$$

Update the inertia weight by following equation (10)

$$
Q=Q_{\max }-\left[\frac{Q_{\max }-Q_{\min }}{i t e r_{\max }}\right] \times \text { iter }
$$

where $Q$ is inertia weight, iter and iter $_{\max }$ are the iteration count and maximum iteration, respectively.

4. Update the velocity and position of each particle. For the particle $i$, the updated velocity and position can be determined by following equations

$$
\begin{gathered}
V_{i}(\text { iter }+1)=Q V_{i}(\text { iter })+\alpha 1\left[\gamma_{1 i}\left(X_{p t}-X_{i}(\text { iter })\right)\right]+\alpha_{2}\left[\gamma_{2 i}\left(G-X_{i}(\text { iter })\right)\right] \\
X_{i}(\text { iter }+1)=X_{i}(\text { iter })+V_{i}(\text { iter }+1)
\end{gathered}
$$

5. Increment iteration for a step

$$
(\mathrm{iter}=\mathrm{iter}+1)
$$

6. Stop if the convergence or stopping criteria (the error variance is zero or within a tolerable limit) are met, otherwise go to step 2.

\section{RESULTS AND DISCUSSION}

The Proposed algorithm is tested with standard image such as "Lena", High resolution DRA Xband SAR images, RADARSAT image data in Xuzhou, Jiangsu province, China, Oil field in Basra Iraq in Persian Gulf with intricacy roads and slander oil pipelines after multi look processing, Hulu Island area located in Liaoning province in China. The proposed algorithm uses threshold range from 0 to 0.7 for PSO optimization. The performance of the proposed algorithm is tested for various levels of noise corruption and compared with standard filters namely Median 
filter, Lee filter, Frost filter, Kuan filter, Statistic Lee filter and gamma filter. Besides, visual inspection the assessment parameters that have been used to evaluate the speckle reduction performance are:

\subsection{Peak Signal to Noise Ratio}

Peak Signal to Noise Ratio can be calculated as,

$$
M S E=\frac{1}{M N} \sum_{i=1}^{M} \sum_{j=1}^{N}\left(R_{i j}-I_{i j}\right)^{2}
$$

Where, I is the input image and $\mathrm{R}$ is recovered image and $\mathrm{M}, \mathrm{N}$ is the size of the test image.

$$
P S N R=10 \log _{10}\left[\frac{g_{\text {max }}^{2}}{M S E}\right]
$$

Where, $g_{\max }=255$, maximum gray level.

\subsection{Noise Mean Value (NMV), Noise Variance (NV)}

NV determines the contents of the speckle in the image. A lower variance gives a "cleaner" image as more speckle is reduced, although, it not necessarily depends on the intensity [17]. The formulas for the NMV, NV and NSD calculation are

$$
\begin{aligned}
& N M V=\frac{1}{R C} \sum_{r=1}^{R} \sum_{c=1}^{C} I_{d}(r, c) \\
& N V=\frac{1}{R C} \sum_{r=1}^{R} \sum_{c=1}^{C}\left(I_{d}(r, c)-N M V\right)^{2}
\end{aligned}
$$

Where R,C is the size of the de-speckled image (Id). On the other hand, the estimated noise variance is used to determine the amount of smoothing needed for each case for all filters.

\subsection{Equivalent Number of Looks (ENL)}

Another good approach of estimating the speckle noise level in a SAR image is to measure the ENL over a uniform image region [18]. A larger value of ENL usually corresponds to a better quantitative performance. The value of ENL also depends on the size of the tested region, theoretically a larger region will produces a higher ENL value than over a smaller region but it also tradeoff the accuracy of the readings. Due to the difficulty in identifying uniform areas in the image, we proposed to divide the image into smaller areas of $25 \times 25$ pixels, obtain the ENL for each of these smaller areas and finally take the average of these ENL values. The formula for the ENL calculation is

$$
E N L=\frac{N M V^{2}}{N S}
$$

The significance of obtaining ENL measurement in this work is to analyze the performance of the filter on the overall region as well as in smaller uniform regions. 
International Journal of Information Sciences and Techniques (IJIST) Vol.2, No.2, March 2012
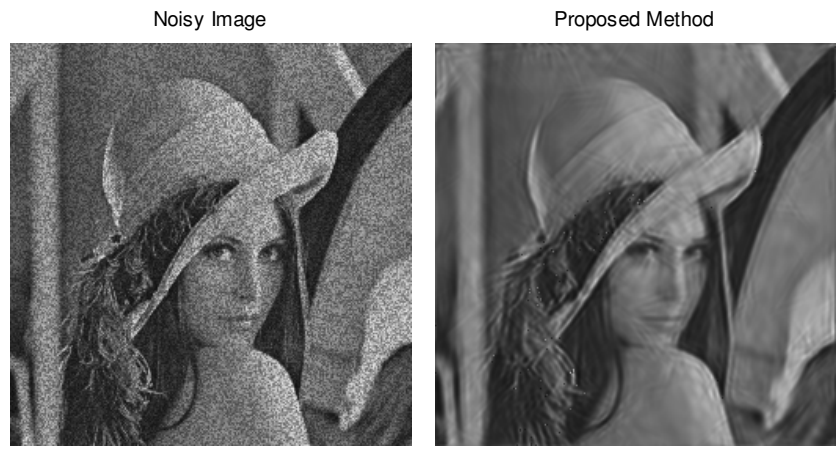

Figure.3 (a)

Figure.3 (b)

Figure.3 (a) Noisy Image corrupted with 0.04 Variance (b) Proposed Method

Table.1 PSNR Measurement for Lena Image

\begin{tabular}{|c|l|l|l|l|l|l|l|l|l|}
\hline $\begin{array}{c}\text { Noise } \\
\text { Variance }\end{array}$ & $\begin{array}{c}\text { Noise } \\
\text { Image }\end{array}$ & $\begin{array}{c}\text { Median } \\
\text { Filter }\end{array}$ & $\begin{array}{c}\text { Kuan } \\
\text { Filter }\end{array}$ & $\begin{array}{c}\text { Lee } \\
\text { Filter }\end{array}$ & $\begin{array}{c}\text { Statistic } \\
\text { Lee }\end{array}$ & $\begin{array}{c}\text { Frost } \\
\text { Filter }\end{array}$ & $\begin{array}{c}\text { GMAP } \\
\text { Filter }\end{array}$ & $\begin{array}{c}\text { Proposed } \\
\text { Method }^{[12]}\end{array}$ & $\begin{array}{c}\text { Proposed } \\
\text { Method }\end{array}$ \\
\hline 0.01 & 27.68 & 30.75 & 27.35 & 33.37 & 28.69 & 31.69 & 29.94 & 33.94 & 34.99 \\
\hline 0.02 & 24.71 & 29.03 & 26.62 & 31.87 & 26.94 & 29.56 & 29.20 & 32.79 & 33.43 \\
\hline 0.03 & 22.94 & 27.83 & 26.01 & 30.76 & 25.68 & 28.11 & 28.57 & 31.51 & 32.08 \\
\hline 0.04 & 21.71 & 26.85 & 25.46 & 29.88 & 24.72 & 27.05 & 28.02 & 30.79 & 31.08 \\
\hline 0.05 & 20.71 & 29.15 & 24.99 & 29.15 & 23.91 & 26.18 & 27.54 & 29.45 & 30.15 \\
\hline
\end{tabular}

Table 1 shows the PSNR measurement of Lena image for various level of noise corruption. From this Table 1 it is inferred that the proposed method gives better result compared to Other filtering techniques such as Lee filter, Kuan filter, Frost filter, gamma MAP filter, etc.

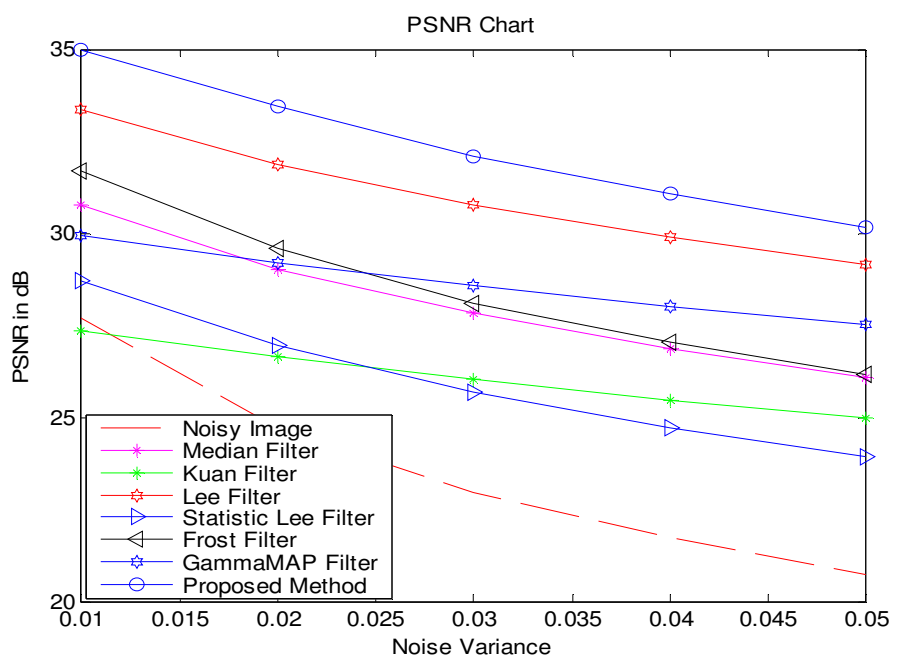

Figure. 4 PSNR Chart for Lena image 


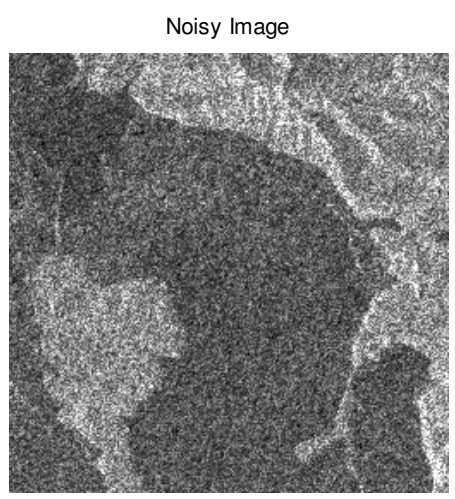

Fig.5.(a)

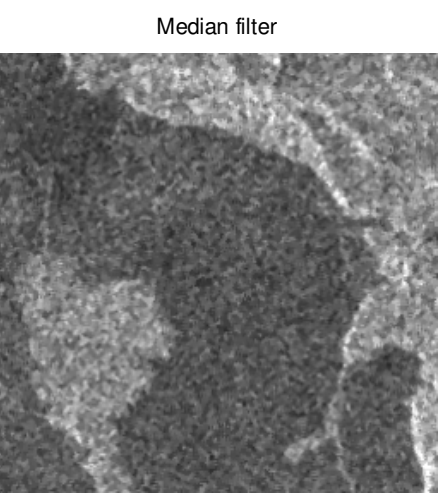

Fig.5.(b)

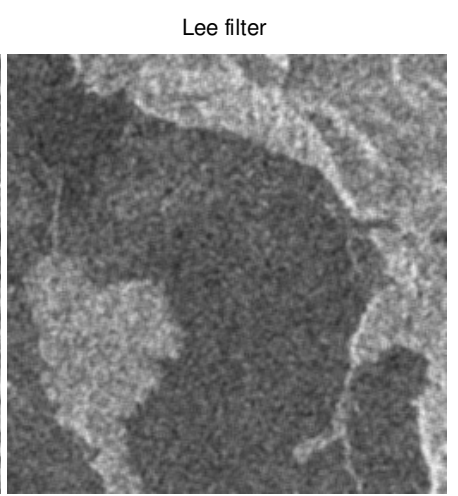

Fig.5.(c)

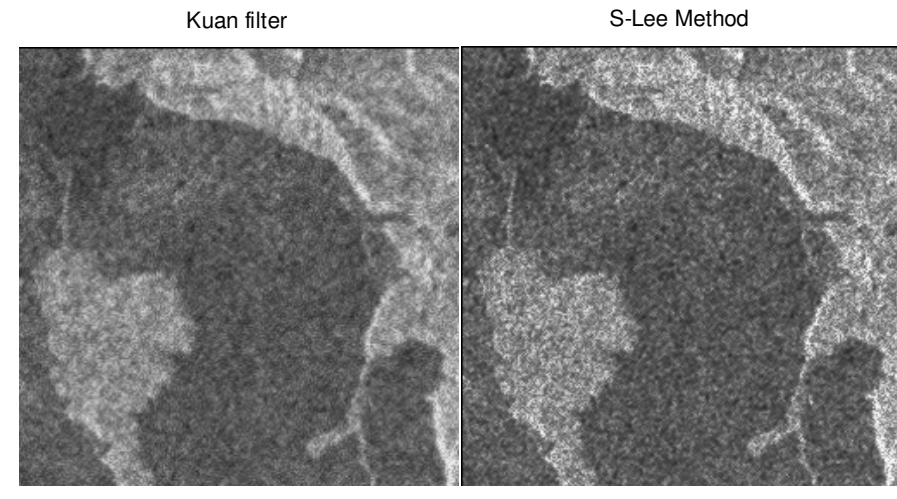

Fig.5.(d)
Fig.5.(e)

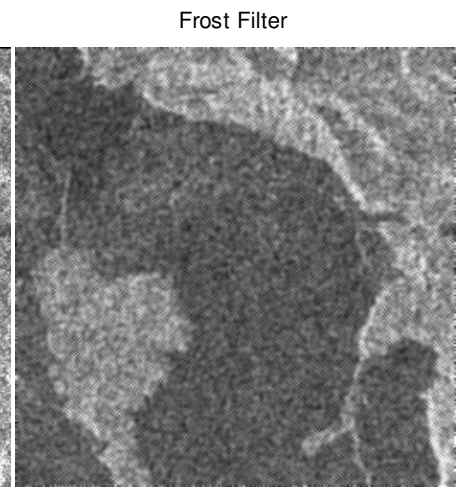

Fig.5.(f)

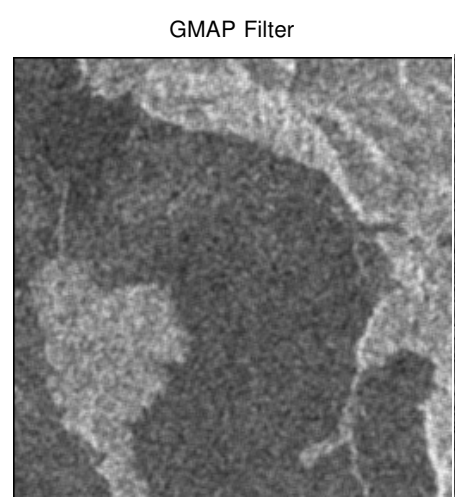

Fig.5.(g)

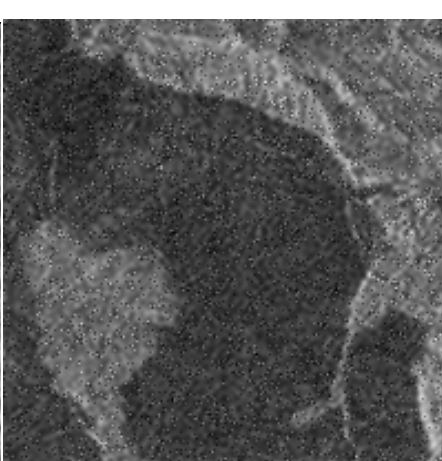

Fig.5.(h)

Proposed Method

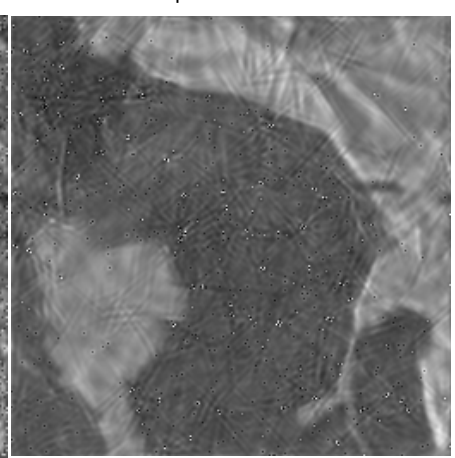

Fig.5.(i)

Figure.5 (a) Hulu Island area SAR Image (b) Median filtered image (c) Lee filter image (d) Kuan filtered image (e)statistical Lee filtered image (f) Frost filtered image (g) GMAP filtered image (h) Proposed Method[12] (i) Proposed Method

Figure 3 is the PSNR chart for the table 1 these values are measure based on reference image. For no reference case Hulu Island area SAR Image in Liaoning province in China has been taken and its assement parameter value is shown in Table 2. 
International Journal of Information Sciences and Techniques (IJIST) Vol.2, No.2, March 2012

Table.2 Assessment Parameter Measurement for SAR images

\begin{tabular}{|l|l|l|c|}
\hline \multirow{2}{*}{ Methods } & \multicolumn{3}{|c|}{$\begin{array}{c}\text { Assessment Parameter } \\
\text { (X-Band Geographical SAR Image) }\end{array}$} \\
\cline { 2 - 4 } & $\begin{array}{c}\text { Noise Mean Value } \\
\text { (NMV) }\end{array}$ & $\begin{array}{c}\text { Noise Variance } \\
\text { (NV) }\end{array}$ & ENL \\
\hline Noisy Image & 61.7744 & 2386.3 & 11.9883 \\
\hline Median Filter & 61.2180 & 2139.3 & 15.9092 \\
\hline Kuan Filter & 61.0802 & 2213.7 & 17.0578 \\
\hline Lee Filter & 61.7501 & 2351.2 & 17.2436 \\
\hline Statistic Lee Filter & 61.4836 & 2269.1 & 15.5767 \\
\hline Frost Filter & 61.1681 & 2121.8 & 16.3475 \\
\hline GMAP Filter & 61.0633 & 2103.9 & 17.2388 \\
\hline $\begin{array}{l}\text { Proposed } \\
\text { Method }^{[12]}\end{array}$ & 60.9391 & 2098.7 & 17.4594 \\
\hline Proposed Method & $\mathbf{6 0 . 3 1 8 1}$ & $\mathbf{2 0 8 0 . 8}$ & $\mathbf{1 7 . 9 6 5 7}$ \\
\hline
\end{tabular}

This proposed Algorithm is tested for various real time SAR image taken at various countries in various climate. Proposed algorithm is applied over some of the SAR images, like X-band SAR image, Oil field of Basra Iraq, RADAR SAT image for analyse.

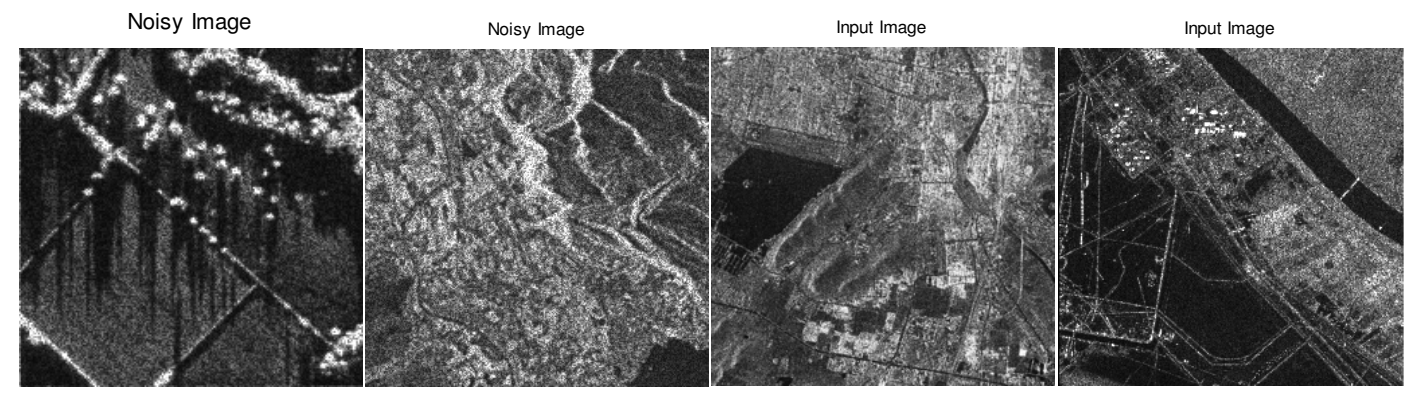

Fig.6. (a)

Fig.6.(b)

Fig.6.(c)

Fig.6.(d)

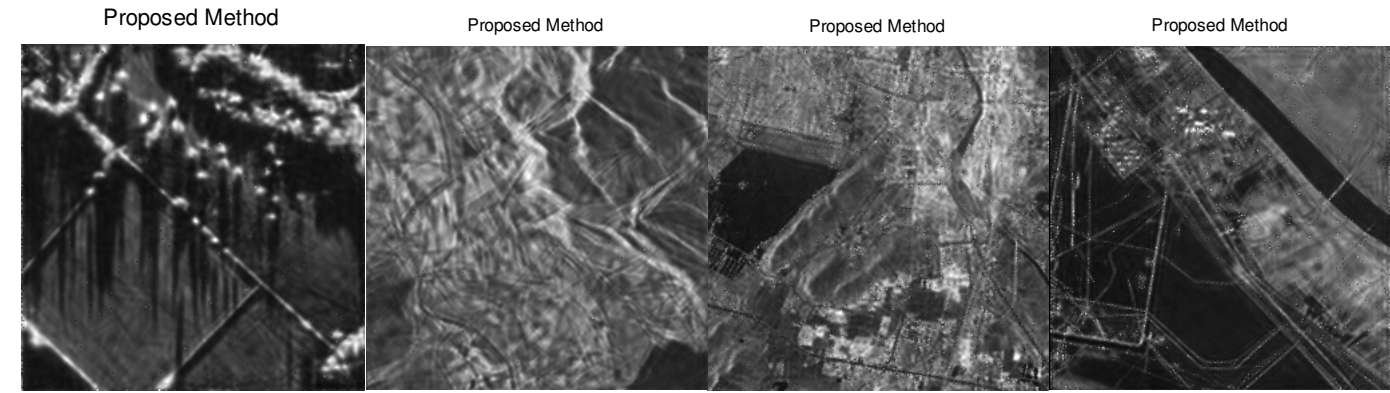

Fig.6. (e)

Fig.6.(f)

Fig.6.(g)

Fig.6.(g)

Figure.6 (a) X-band SAR image(ENL=6.3298) (b)SAR image(ENL= 5.2805) (c)RADAR SAT image $(E N L=2.9178)(d)$ Oil field of Basra Iraq(ENL=5.8487) (e)Despeckled Image of (a) 15.9879) (g)Despeckled Image of $(\mathrm{b})(\mathrm{ENL}=10.6107) \quad(\mathrm{g})$ Despeckled Image of $(\mathrm{c})(\mathrm{ENL}=5.3918)$ (h)Despeckled Image of $(\mathrm{d})(\mathrm{ENL}=18.4895)$ 
International Journal of Information Sciences and Techniques (IJIST) Vol.2, No.2, March 2012

Figure 6 depicts various SAR images and their corresponding despeckled image with their ENL. Higher ENL value shows that the despeckled image contains lesser speckle noise.

\section{CONCLUSION}

This paper illustrates that the proposed curvelet based despeckling algorithm using PSO performs much better in several aspects than other wavelet based method and filtering technique. Particle Swarm Optimization technique is used to minimize the Average Power Spectrum Value of uniform block. Average power spectrum Value measured in the homogenous block defines the amount of noise present in the image. Noise will be more, when APSV value is maximum. The proposed algorithm thus achieves better despeckling performance and edge preservation capability. Experimental results using real SAR images demonstrate that the proposed method can reduce the speckle to a great extent while preserving texture and strong radiometric scatter points.

\section{REFERENCES}

[1] Tinku Acharya and Ajoy K. Ray, "Image Processing Principles and Applications", 2005 edition A John Wiley \& Sons, Mc., Publication.

[2] Rafael C. Gonzalez and Richard E. Woods, "Digital Image Processing", Second Edition, Pearson Education.

[3] J.W.Goodman, "Some fundamental properties of speckle," J. Opt.Soc.Amer. vol.66, no. 11, pp.11451150, Nov 1976.

[4] Cheng Zhili, Wang Hongxia,Luo Yong, "Wavelet's theory and Application" Beijing:Science Press, 2004.

[5] Jiang,Zhao, “ Improved image denoising method based on Curvelet Transform:, IEEE trans. June 20232010.

[6] J.S.Lee, "Digital image enhancement and noise filtering by use of local statistics", IEEE Trans. On Pattern Analysis and Matching Intelligence, vol. PAMI-2, pp.165-168, 1980.

[7] J.S.Lee, "Refined filtering of image noise using local statistics" Computer Graphic and Image Processing 15, 380-389 (1981)

[8] D.T.Kuan et al., "Adaptive restoration of images with speckle”, IEEE Trans. Acc. Speech and signal Proc. Vol. 35, no.3 pp.373-383, March 1987

[9] V.S.Frost et al, "A model for radar images and its application to adaptive digital filtering of multiplicative noise”, IEEE Trans. Pattern Anal. And Machine Intell.,vol. PAMI-4, pp.157-166. 1982.

[10] A. Lopes et al., "Adaptive speckle filters and Scene heterogeneity", IEEE Transaction on Geoscience and Remote Sensing, Vol. 28, No. 6, pp. 992- 1000, Nov. 1990.

[11] Jannes R. Sveinsson and Jon Atli Benediktsson, "Speckle Reduction and Enhancement of SAR images in the wavelet domain," Geoscience and Remote Sensing Symposium IGARSS'96,vol.1, pp.63-66,Mat 1996.

[12] S. Mohamed Mansoor Roomi1, D. Kalaiyarasi, D. Sabarinathan and R. Abitha "Curvelet Transform Based Despeckling for SAR Images using Particle Swarm Optimization Technique”, Page 217-222, NCSIP'12, 2012. 
International Journal of Information Sciences and Techniques (IJIST) Vol.2, No.2, March 2012

[13] E.J.Candes, "Monoscale Ridgelets for the representation of image with Edges",Dept. Statistics, Standford univerisity, standford,CA,1999,1-26.

[14] D.L.Donoho,A.G.Flesia,"Digital Ridgelet tranform based on true ridge functions. J.stoeckler,G.V.Welland. Beyond wavelets, pittsburgh,PA,USA:Academic press,2002.1-33.

[15] J. Kennedy and R. Eberhart, "Particle swarm optimization," IEEE International Conference on Neural Networks, Vol. 4, 1995, 1942-1948.

[16] Emmanuel Cand`es, Laurent Demanet, David Donoho and Lexing Ying, "Fast Discrete Curvelet Transforms", Dept of Statistic,Standford univeristy,2005

[17] Ashkan Masoomi, Mahmood Godratiyan "Remove speckle of SAR Images by Directional smoothing of wavelet coefficients" New Aspects Of Signal Processing, Computational Geometry And Artificial Vision,2008.

[18] Guozhong Chen, Xingzhao Liu "Contourlet-based Despeckling for SAR Image Using Hidden Markov Tree and Gaussian Markov Models” IEEE Transaction, 2007.

[19] Yan Zhang, Ping An, Qiuwen Zhang, Liquan Shen, Zhaoyang Zhang, "A No-Reference Image Quality Evaluation Based On Power Spectrum”, IEEE Transaction,2011.

[20] Biao Hou, Honghua Liu, Licheng Jiao,'The Despeckling of SAR images on the curvelet transform”, IEEE Transaction, APSR,534170,2009.

\section{Authors}

S.Md Mansoor Roomi Received his B.E degree in Electronic and Communication Engineering from Madurai Kamarajar University in 1990 and M.E (Power System) and M.E(Communication systems) from Thiagarajar College Engineering Madurai in 1992 \&1997 and PhD in 2009 from Madurai Kamaraj University. His Primary interests include Image enhancement and Analysis

D.Kalaiyarasi received her B.E degree in Electronics and communication Engineering from Madurai Thiagarajar college of Engineering in 2006 and Doing M.E (communication Systems) in Madurai Thiagarajar College of Engineering. Her Primary interests include Image Enhancement and Analysis.
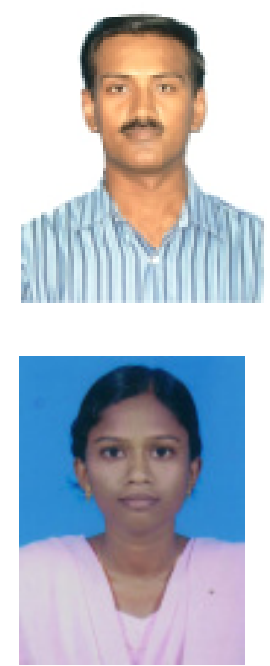